\title{
POTENCIAL REPRODUTIVO E DE SOBREVIVÊNCIA DE Podisus nigrispinus DALLAS (HETEROPTERA: PENTATOMIDAE) SOBRE Thyrinteina arnobia STOLL (LEPIDOPTERA: GEOMETRIDAE) E Tenebrio molitor L. (COLEOPTERA: TENEBRIONIDAE)
}

\author{
Anderson Mathias Holtz*, José Cola Zanuncio**, Claudinei Lima Oliveira***, Dirceu Pratissoli*, \\ Angelo Pallini****, Jeanne Scardini Marinho***, Ulysses Rodrigues Vianna*** \\ *Eng. Agrônomo, Dr., UFES - aholtz@insecta.ufv.br; pratissoli@cca.ufes.br \\ **Eng. Florestal, Ph.D., UFV - zanuncio@ufv.br \\ ***Eng. Florestal, M.Sc., UFV - claudinei@insecta.ufv.br - jeanne@insecta.ufv.br - ulyssesvianna@insecta.ufv.br \\ ****Eng. Agrônomo, Ph.D., UFV - pallini@ufv.br
}

Recebido para publicação: 14/03/2006 - Aceito para publicação: 30/10/2006

\begin{abstract}
Resumo
O gênero Eucalyptus abrange as espécies arbóreas mais utilizadas para reflorestamento no Brasil. Devido ao seu elevado conteúdo de substâncias secundárias, esperava-se que os insetos não causassem sérios danos ao eucalipto. Contudo, muitos insetos, principalmente os da ordem Lepidoptera, tornaram-se sérias pragas na eucaliptocultura. O predador Podisus nigrispinus Dallas (Heteroptera: Pentatomidae) vem sendo utilizado contra insetos herbívoros tais como Thyrinteina arnobia Stoll (Lepidoptera: Geometridae), principal praga do eucalipto, mas não se têm dados sobre seu estabelecimento em áreas de eucalipto. Dessa forma, o objetivo deste trabalho foi avaliar o potencial reprodutivo e sobrevivência de $P$. nigrispinus sobre $T$. arnobia proveniente de eucalipto e larvas de Tenebrio molitor L. (Coleoptera: Tenebrionidae) provenientes de criação massal em laboratório. Os aspectos biológicos de P. nigrispinus foram melhores sobre pupas de T. molitor em comparação aos indivíduos criados sobre lagartas de T. arnobia. Provavelmente, as lagartas de $T$. arnobia estão seqüestrando as substâncias secundárias das plantas de eucalipto e utilizando em sua defesa contra predadores como $P$. Nigrispinus, e se estes inimigos naturais não estiverem adaptados a tais compostos, a sua performance no controle da praga e no seu próprio desenvolvimento podem ser afetados negativamente.

Palavras-chave: Predador; controle biológico; Eucalyptus; herbívoro.
\end{abstract}

\begin{abstract}
Biological aspects of Podisus nigrispinus Dallas (Heteroptera: pentatomidae) on Thyrinteina arnobia stoll (Lepidoptera: geometridae) and Tenebrio molitor L. (Coleoptera: tenebrionidae). The genus Eucalyptus contains the arboreal species most used to reforestation in the Brazil. Like the plants of this genus presents elevated content of secundarity substances, was waited that they might not cause serious damages to the eucalyptus. However, many insects, principally those of the order Lepidoptera, becomes serious pests in the culture of eucalyptus. The predator Podisus nigrispinus Dallas (Heteroptera: Pentatomidae) has been used against herbivorous insects like Thyrinteina arnobia Stoll (Lepidoptera: Geometridae), but there is not data about your establishment in eucalyptus areas. The objective of this work was evaluate the reproductive potential and to perform a analyses of survival of P. nigrispinus on $\mathrm{T}$. arnobia proceeding of eucalyptus and grub Tenebrio molitor L. (Coleoptera: Tenebrionidae) proceeding of mass rearing in laboratory. The biologics aspects of $\mathrm{P}$. nigrispinus were better on pupa of $T$. molitor when compared the individuals create on caterpillar of $T$. arnobia. Probably, the caterpillar of T. arnobia are kidnapped the secundarity substances from the Eucalyptus plants and using for your defense against predators like P. nigrispinus and, if these naturals enemies are not adapted to these substances, their performance in the pest control and on their own development may be negatively affected.

Keywords: Predator; biological control; Eucalyptus; herbivory.
\end{abstract}




\section{INTRODUÇÃO}

A atividade florestal no Brasil é muito recente, pois somente a partir de 1966, com a lei de incentivos fiscais para reflorestamento, é que esse setor começou a tomar impulso (ZANUNCIO et al., 1995; SANTOS et al., 2000). Dentre as espécies utilizadas para o reflorestamento, destacam-se as do gênero Eucalyptus (Myrtaceae), planta exótica proveniente da Austrália (ZANUNCIO et al., 1993).

Devido à presença de altas concentrações de compostos secundários nas plantas de Eucalyptus, os pesquisadores acreditavam que insetos não causariam sérios danos a essa espécie. Entretanto, há relatos de insetos da ordem Lepidoptera, como, por exemplo, Thyrinteina arnobia Stoll (Lepidoptera: Geometridae), danificando plantas de eucalipto (ANJOS et al., 1986; BRAGANÇA et al., 1998).

Segundo Koptur (1985) e Hill (1987), a incidência de insetos herbívoros sobre plantas afeta o desenvolvimento natural das árvores, por interferir na taxa e no equilíbrio dos processos fisiológicos internos, o que pode resultar na paralisação do crescimento e/ou morte das árvores.

De acordo com Zanuncio et al. (1994), o método de controle mais utilizado contra herbívoros em plantios de eucalipto, até então, é o químico. No entanto, este traz uma série de desvantagens ao ambiente, podendo inclusive ser letal aos inimigos naturais desses desfolhadores. No Brasil, como método de controle alternativo, espécies de predadores da família Pentatomidae vêm sendo usadas em surtos de lagartas desfolhadoras em eucaliptais, tais como Brontoris tabidus Signoret, Supputius cincticeps (Stall) e Podisus nigrispinus Dallas (Heteroptera: Pentatomidae), por sua facilidade de criação massal em laboratório.

Entretanto, a criação desses predadores em laboratório, sob dietas artificiais, facilitam e diminuem os custos de produção de inimigos naturais, mas podem comprometer a eficiência deles em campo, pois os estímulos a que estão submetidos numa criação massal são consideravelmente diferentes daqueles encontrados em presas naturais, podendo estes afetar negativamente os aspectos biológicos de predadores e parasitóides (SZNAJDER; HARVEY, 2003).

Dessa forma, o objetivo deste trabalho foi estudar os aspectos biológicos de $P$. nigrispinus sobre lagartas de T. arnobia criadas com folhas de eucalipto e pupas de Tenebrio molitor L. (Coleoptera: Tenebrionidae) provenientes de criação massal em laboratório.

\section{MATERIAL E MÉTODOS}

\section{Instalações, criação e manutenção de $T$. arnobia}

Os adultos de T. arnobia, provenientes de coleta em plantios de eucalipto no estado de Minas Gerais, foram levados para o laboratório de Acarologia da Universidade Federal de Viçosa (UFV) e mantidos em temperatura de $25 \pm 2{ }^{\circ} \mathrm{C}$, fotofase de 12 horas e umidade relativa de $70 \pm 10 \%$. Casais de $T$. arnobia foram individualizados e colocados em potes plásticos $(500 \mathrm{ml})$ com tampa plástica, nas quais havia um orifício central, vedado com tela de malha fina, tipo organza, para permitir a aeração no recipiente. Nos potes, foram colocadas tiras de papel presas à tampa para que nelas fossem efetuadas as posturas. Logo após a eclosão dos ovos, as lagartas de $T$. arnobia foram colocadas em sacos constituídos por tecido tipo organza $(0,70 \times 0,40 \mathrm{~m})$ envolvendo galhos de plantas de Eucalyptus cloesiana F. Muell sem danos. Nos primeiros estádios, as lagartas foram removidas para outros galhos com sacos de organza, quando as folhas começavam a secar. A troca de galhos foi mais freqüente a partir do quarto estádio, período em que as lagartas alimentam-se mais vorazmente.

\section{Criação de $P$. nigrispinus e desenvolvimento dos experimentos}

Os testes com $P$. nigrispinus, foram conduzidos no laboratório de Acarologia da UFV, em Viçosa, Minas Gerais, sob condições de laboratório $\left(25 \pm 2^{\circ} \mathrm{C}, 70 \pm 10 \%\right.$ de U.R. e fotoperíodo de $\left.12 \mathrm{~h}\right)$, em lagartas de T. arnobia criadas em E. cloesiana e em pupas de T. molitor.

Ninfas de primeiro estádio de $P$. nigrispinus foram obtidas da criação massal do Laboratório de Entomologia Florestal do Departamento de Biologia Animal da UFV. As ninfas foram criadas sob as mesmas condições laboratoriais, do primeiro estádio à fase adulta, em grupos de 5 indivíduos em placas de Petri $(15,0 \times 1,2 \mathrm{~cm})$ contendo um chumaço de algodão no seu interior, o qual era diariamente umedecido com água destilada. A alimentação desses insetos foi realizada seguindo-se a metodologia de Zanuncio et al. (1994), em dieta com pupas de T. molitor.

No início da fase adulta, esses percevejos foram sexados pela aparência externa da genitália e tamanho do corpo. No primeiro dia após passarem para a fase adulta, os indivíduos foram agrupados em 
casais, colocando-se um casal por placa de Petri (conforme descrição anterior), e divididos em dois grupos. O tratamento um foi alimentado com lagartas de $T$. arnobia de $5^{\circ}$ ou $6^{\circ}$ instar, provenientes de $E$. Cloeziana, e no tratamento dois P. nigrispinus continuou sendo alimentado com pupas de $T$. molitor, sendo oferecida diariamente uma lagarta de T. arnobia e uma pupa de T. molitor por placa de Petri, respectivamente, para os tratamentos um e dois. Foram realizadas 25 repetições para cada tratamento.

Foram observados o número de posturas e o número de ovos, além da taxa de sobrevivência de machos e fêmeas de $P$. nigrispinus. As posturas foram retiradas das placas de Petri e colocadas em placas de Petri menores $(9,0 \times 1,2 \mathrm{~cm})$ para se observar a viabilidade dos ovos e o número de ninfas.

Os dados foram analisados pelo Teste $\mathrm{F}$ e os modelos da análise de sobrevivência foram ajustados pelo módulo de equações não-lineares (método de estimativa de parâmetros quasi-newton e Hooke-Jeeves pattern moves) (StatSoft 1995): $\mathrm{S}(\mathrm{t})=\exp (\mathrm{t} / \mathrm{b}) \wedge \mathrm{c}$, onde $\mathrm{S}$ é a probabilidade de sobrevivência no tempo t; $t$ é o tempo; b e c são os parâmetros de escala e forma, respectivamente. Os modelos foram analisados pelo teste de identidade de modelo L\&O.

\section{RESULTADOS}

Analisando-se os parâmetros reprodutivos e de sobrevivência de $P$. nigrispinus criados em pupas de $T$. molitor e lagartas de $T$ arnobia, observou-se diferença significativa $(\mathrm{P}<0,05)$ entre os tratamentos para a maioria dos parâmetros testados. Apenas no parâmetro viabilidade dos ovos não foi observada diferença significativa $(\mathrm{P}>0,05)$ (Tabela 1$)$

Tabela 1. Aspectos biológicos de P. nigrispinus (Heteroptera: Pentatomidae) criados sobre pupas de $T$. molitor (Coleoptera: Tenebrionidae) e lagartas de T. arnobia (Lepidoptera: Geometridae).

Table 1. Biological Aspects of the P. nigrispinus (Heteroptera: Pentatomidae) fed on pupas of the $T$. molitor (Coleoptera: Tenebrionidae) and caterpillars of the T. arnobia (Lepidoptera: Geometridae).

\begin{tabular}{lcc}
\hline Parâmetros & Tenebrio molitor & Thyrinteina arnobia \\
\hline Número de postura/fêmea & $14,94 \pm 7,17 \mathrm{a}$ & $2,44 \pm 1,39 \mathrm{~b}$ \\
Número de ovos/ fêmea & $271,06 \pm 38,46 \mathrm{a}$ & $52,56 \pm 11,72 \mathrm{~b}$ \\
Número de ninfas/fêmea & $245,75 \pm 52,71 \mathrm{a}$ & $48,81 \pm 17,01 \mathrm{~b}$ \\
Viabilidade (\%) & $90,41 \pm 8,32 \mathrm{a}$ & $92,87 \pm 5,87 \mathrm{a}$ \\
\hline
\end{tabular}

Médias seguidas de uma mesma letra nas linhas não diferem entre si, pelo teste $\mathrm{F}$, ao nível de $5 \%$ de significância.

$\mathrm{O}$ número de posturas foi significativamente maior $(\mathrm{P}<0,05)$ quando $P$. nigrispinus foi alimentado com $T$. molitor $(14,94)$ do que fêmeas criadas com $T$. arnobia $(2,44)$ (Tabela 1). A mesma tendência foi observada para o número médio de ovos por oviposição (Figura 1).

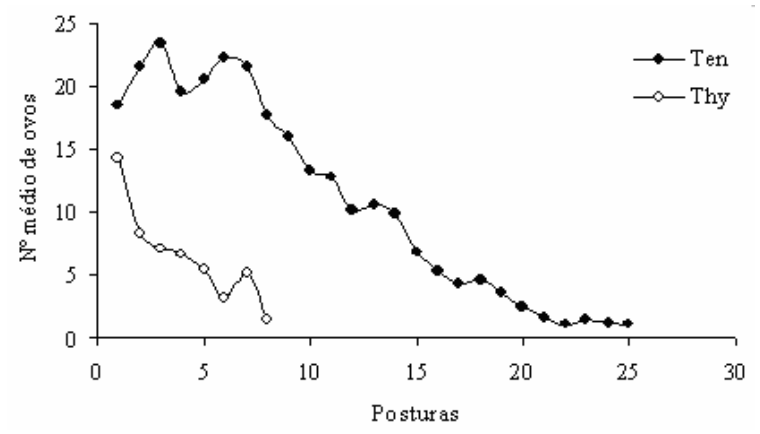

Figura 1. Número de ovos/postura de P. nigrispinus (Heteroptera: Pentatomidae) criados sobre pupas de T. molitor (Coleoptera: Tenebrionidae) (Ten) e lagartas de T. arnobia (Lepidoptera: Geometridae) (Thy).

Figure 1. Number of the eggs/oviposition of the P. nigrispinus (Heteroptera: Pentatomidae) fed on pupas of the T. molitor (Coleoptera: Tenebrionidae) (Ten) and caterpillars of the T. arnobia (Lepidoptera: Geometridae) (Thy). 
$\mathrm{O}$ número de ovos/fêmea foi significativamente maior $(\mathrm{P}<0,05)$ em fêmeas de $P$. nigrispinus criadas com T. molitor $(271,06)$, quando comparado com o de fêmeas criadas com T. arnobia $(52,56)$ (Tabela 1). O número médio de ninfas/fêmea também foi significativamente maior $(\mathrm{P}<0,05)$ para fêmeas de $P$. nigrispinus criadas com o T. molitor $(245,75)$, quando comparado com o de fêmeas criadas com $T$. arnobia $(48,81)$ (Tabela 1).

A longevidade média de $P$. nigrispinus foi maior $(\mathrm{P}<0,05)$ quando este predador foi alimentado com T. molitor (36 dias para machos e 35 dias para fêmeas), se comparada à dos alimentados com lagartas de T. arnobia (16 dias para machos e 15 dias para fêmeas) (Figura 2).

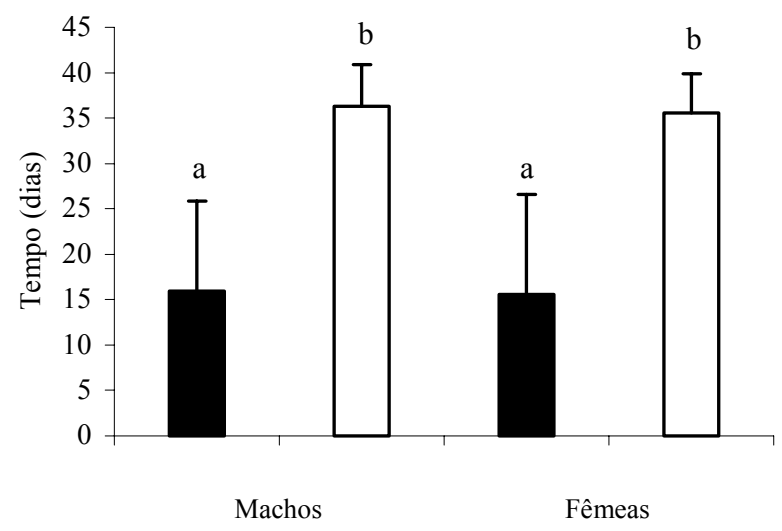

Figura 2: Sobrevivência (dias) de adultos de P. nigrispinus (Heteroptera: Pentatomidae) criados sobre pupas de T. molitor (Coleoptera: Tenebrionidae) ( $\square$ ) e lagartas de T. arnobia (Lepidoptera: Geometridae) (ם). Médias com letras diferentes diferem entre si, pelo teste F, a 5\% de significância.

Figure 2: Survival of adults of $P$. nigrispinus (Heteroptera: Pentatomidae) fed on pupas of the T. molitor (Coleoptera: Tenebrionidae) ( $\square$ ) and caterpillars of the T. arnobia (Lepidoptera: Geometridae) (घ). Averages with different letters differ to each other, for the test $\mathrm{F}$, to $5 \%$ of the significance.

A curva de sobrevivência de adultos de P. nigrispinus (Figura 3) mostra sobrevivência aproximada de $90 \%$ em torno dos 30 dias para esse predador criado em pupas de T. molitor e de $18 \%$ para indivíduos criados em lagartas de T. arnobia (Figura 3).

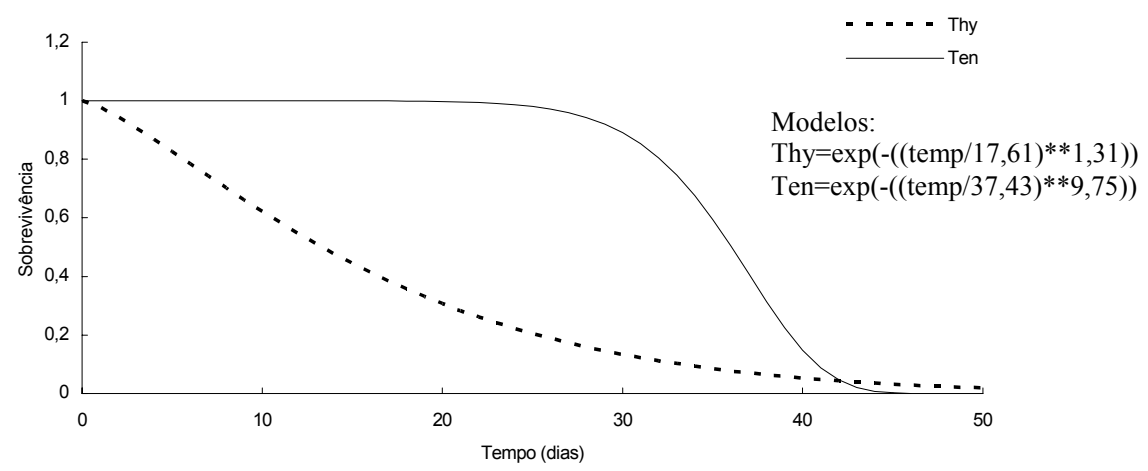

Figura 3. Curva de sobrevivência de P. nigrispinus (Heteroptera: Pentatomidae) criados sobre pupas de T. molitor (Coleoptera: Tenebrionidae) (Ten) e lagartas de T. arnobia (Lepidoptera: Geometridae) (Thy).

Figure 3. Curves of survival of P. nigrispinus (Heteroptera: Pentatomidae) fed on pupas of the T. molitor (Coleoptera: Tenebrionidae) (Ten) and caterpillars of the $T$. arnobia (Lepidoptera: Geometridae) (Thy). 


\section{DISCUSSÃO}

Lagartas de T. arnobia provenientes de eucalipto diminuem o potencial reprodutivo e de sobrevivência de $P$. nigrispinus em relação àqueles criados com pupas de T. molitor. Segundo Sznajder; Harvey (2003), o efeito das plantas na qualidade da presa e/ou hospedeiro, e subseqüentemente no desenvolvimento de inimigos naturais, pode ser devido à presença de aleloquímicos no tecido hospedeiro via seqüestro de compostos.

As plantas de eucalipto apresentam altas concentrações de compostos secundários, como taninos, fenóis e óleos essenciais (OHMART; EDWARDS, 1991). De acordo com Morais et al. (1998), os eucaliptos atacados por herbívoros recebem um estímulo fisiológico para produção de composto de defesa. Porém, os herbívoros, em contra-resposta, podem seqüestrar os compostos secundários e utilizálos em seu próprio benefício, atuando negativamente na performance de populações de inimigos naturais (TRIGO, 2000; VLIEGER et al., 2004). Schenk; Bacher (2002) e Muller; Brakefield (2003) argumentam que insetos herbívoros que não apresentam esse tipo de proteção química podem ser altamente afetados pelos seus inimigos naturais.

Dessa forma, herbívoros que se alimentam de folhas de eucalipto poderiam estar seqüestrando os compostos tóxicos dessas plantas, tornando-se impalatáveis ou tóxicos aos inimigos naturais. Como a eucaliptocultura é relativamente recente no Brasil, em termos evolutivos, os inimigos naturais teriam dificuldade em assimilar os possíveis compostos secundários seqüestrados pelas lagartas, sendo, então, afetados negativamente no ato da predação. Oliveira (2001) demonstrou que espécies de parasitóides do gênero Trichogramma não parasitaram ovos de T. arnobia, além de apresentarem mortalidade de $100 \%$ após 24 horas de contato com os ovos do hospedeiro. Porém, apresentam melhor performance quando os ovos desse herbívoro são lavados com uma solução de xilol a $0,1 \%$. Isso indica que esse herbívoro, provavelmente, seqüestra substâncias químicas das plantas de eucalipto e as deposita sobre seus ovos. Segundo Nishida (2002), herbívoros seqüestram compostos secundários como estratégia de defesa contra inimigos naturais e, também, para proteção nas fases mais vulneráveis, como a postura.

Weiser e Stamp (1998) mostraram que a adição de aleloquímicos, retirados de plantas de tomate, em uma dieta artificial para Manduca sexta L. (Lepidoptera: Sphingidae), causou efeito negativo sobre a performance e desenvolvimento do predador Podisus maculiventris Say (Heteroptera: Pentatomidae). No trabalho realizado por Montillor et al. (1991), foi observado que a vespa Mischocyttarus flavitarsis (Saussure) (Hymenoptera: Vespidae) foi afetada negativamente por alcalóides seqüestrados por lagartas de suas plantas hospedeiras.

T. arnobia é considerado o principal lepidóptero-praga em plantios de eucalipto, devido aos seus surtos periódicos e danos causados aos plantios dessa essência florestal (ZANUNCIO et al., 1991; SANTOS et al., 1996). Em trabalhos realizados por Holtz et al. (2003a, b), observou-se que o desempenho de T. arnobia foi melhor em plantas de Eucalyptus spp. do que em plantas de Psidium guajava L. (goiaba). Dessa forma, observa-se que esse herbívoro está mais especializado em plantas de eucalipto do que em plantas de goiaba. De acordo com Dobler (2001), uma óbvia pré-condição para o seqüestro de compostos secundários de plantas está na freqüência com que determinado herbívoro se alimenta da planta, pois insetos herbívoros especializados em uma determinada espécie de planta são mais passíveis de seqüestrarem compostos que insetos herbívoros generalistas. Segundo Renwick; Lopez (1999), Roessingh et al. (2000) e Stadler (2000), os insetos herbívoros especialistas são conhecidos por exibirem adaptações aos compostos secundários e até mesmo por utilizarem esses compostos como estimulantes na sua alimentação.

Vários estudos têm relatado que aleloquímicos em presas e/ou hospedeiros afetam negativamente o crescimento, desenvolvimento, sobrevivência e morfologia de predadores e parasitóides (DUFFEY; STOUT, 1996; GUNASENA et al., 1990; PARADISE; STAMPE, 1993; HAVILL; RAFFA, 2000). Contudo, em muitos casos esses efeitos são mais freqüentes em inimigos naturais generalistas, enquanto que os inimigos naturais especialistas estão, aparentemente, mais adaptados às toxinas das plantas (BARBOSA et al., 1991; PARADISE; STAMP, 1993; VINSON, 1999; HARVEY et al., 2003). Dessa forma, por ser $P$. nigrispinus um predador generalista e com curta história co-evolutiva com plantas de eucalipto, estaria exposto aos compostos secundários dessa essência florestal. 


\section{CONCLUSÃO}

Os resultados obtidos no presente trabalho permitem concluir que o predador $P$. nigrispinus foi afetado por lagartas de $T$. arnobia provenientes de E. cloesiana, provavelmente, por não estar adaptado aos compostos secundários dessa essência florestal, devido à curta história co-evolutiva entre esses dois organismos (planta e predador). Dessa forma, compostos de defesa da planta que inicialmente deveriam agir no seu benefício, podem afetar negativamente populações de inimigos naturais.

\section{AGRADECIMENTOS}

Ao Conselho Nacional de Desenvolvimento Científico e Tecnológico (CNPq), à Coordenação de Aperfeiçoamento de Pessoal de Nível Superior (CAPES) e à Fundação de Amparo à Pesquisa do Estado de Minas Gerais (FAPEMIG), pela concessão de bolsas.

\section{REFERÊNCIAS}

ANJOS, N.; SANTOS, G. P.; ZANUNCIO, J. C. Pragas do eucalipto e seu controle. Informe Agropecuário, Belo Horizonte, v. 12, p. 50-58, 1986.

BARBOSA, P.; GROSS, P.; KEMPER, J. Influence of plant allelochemicals on the performance of the tobacco hornworm and its parasitoid Cotesia congregata. Ecology, Tempe, v. 72, p. 1567-1575, 1991.

BRAGANCA, M. A. L.; ZANUNCIO, J. C.; PICANÇO, M.; LARANJEIRO, A. J. Effects of environmental heterogeneity on Lepidoptera and Hymenoptera populations in Eucalyptus plantations in Brazil. Forest Ecology and Management, Washington, D.C., v. 103, p. 287-292, 1998.

MORAIS, C. M.; LEWIS, W. J.; PARE, P. W.; ALBORN, H.T.; TUMLINSON, J. H. Herbivore-infested plants selectively attract parasitoids. Nature, London, v. 393, p. 570-573, 1998.

DOBLER, S. Evolutionary aspects of defense by recycled plant compounds in herbivorous insects. Basic and Applied Ecology, Jena, v. 2, p. 15-26, 2001.

DUFFEY, S. S.; STOUT, M. J. Antinutritive and toxic components of plant defence against insects. Archive of Insect Biochemistry and Physiology, New York, v. 32, p. 3-37, 1996.

GUNASENA, G. H.; VINSON, S. B.; WILLIAMS, H. J. Effects of nicotine on growth, development and survival of the tobacco budworm (Lepidoptera: Noctuidae) and the parasitoid Campoletis sonorensis (Hymenoptera: Ichneumonidae). Journal of Economic Entomology, Riverside, v. 83, p. 1777-1782, 1990.

HARVEY, J. A.; VAN DAM, N. M.; GOLS, R. Interactions over four trophic levels: foodplant quality affects development of a hyperparasitoid as mediated through a herbivore and its primary parasitoid. Journal of Animal Ecology, Oxford, GB, v. 72, p. 520-531, 2003.

HAVILL, N. P.; RAFFA, K. F. Compound effects of induced plant responses on insect herbivores and parasitoids: implications for tritrophic interactions. Ecological Entomology, London, v. 25, p. 171-179, 2000 .

HILL, W. E. Polyphenols in the leaves of Eucalyptus L'Herit a chemotaxonomics survey: Introduction and study of the series globulares. Phytoc., v. 5, p. 1075-1090, 1987. Não consegui descobrir o título do periódico, falta local e título por extenso.

HOLTZ, A. M.; OLIVEIRA, H. G.; PALLINI, A.; MARINHO, J. S.; ZANUNCIO, J. C.; OLIVEIRA, C. L. Adaptação de Thyrinteina arnobia em novo hospedeiro e defesa induzida por herbívoros em eucalipto. Pesquisa Agropecuária Brasileira, Brasília, D.F., v. 38, p. 453-458, 2003a.

HOLTZ, A. M.; OLIVEIRA, H. G.; PALLINI, A.; VENZON, M.; ZANUNCIO, J. C.; OLIVEIRA, C. L.; MARINHO, J. S.; ROSADO, M. da C. Desempenho de Thyrinteina arnobia Stoll (Lepidoptera: Geometridae) em eucalipto e goiaba: o hospedeiro nativo não é um bom hospedeiro? Neotropical Entomology, Londrina, v. 32, p. 427-431, 2003 b. 
KOPTUR, S. Alternative defenses against herbivores in Inga (Fabaceae: Mimosoideae) over na elevational gradient. Ecology, Tempe, v. 66, p. 1639-1650, 1985.

MONTILLOR, C. B.; BERNAYS, E. A.; CORNELIUS, M. L. Responses of two hymenopteran predators to surface chemistry of their prey: significance for an alkaloid-sequestering caterpillar. Journal of Chemical Ecology, New York, v.17, p. 391-399, 1991.

MÜLLER, C.; BRAKEFIELD, P. M. Analysis of a chemical defense in sawfly larvae: easy bleeding targets predatory wasps in late summer. Journal of Chemical Ecology, New York, v. 29, p. 2683-2694, 2003.

NISHIDA, R. Sequestration of defensive substances from plants by Lepidoptera. Annual Review of Entomology, Stanford, v. 47, p. 57-92, 2002.

OHMART, C. P.; EDWARDS, P. B. Insect herbivory on Eucalyptus. Annual Review of Entomology, Stanford, v. 36, p. 637-657, 1991.

OLIVEIRA, H. N. Potencial de uso de Trichogramma como agente de controle biológico de lepidópteros desfolhadores de Eucalyptus e sua associação com percevejos predadores. 80p. Dissertação (Doutorado em Entomologia) - Universidade Federal de Viçosa, Viçosa, MG, 2001.

PARADISE, C. J.; STAMP, N. E. Episodes of unpalatable prey reduce consumption and growth of juvenile praying mantids. Journal of Insect Behavior, New York, v. 6, p. 155-166, 1993.

RENWICK, J. A. A.; LOPEZ, K. Experience-based food consumption by larvae of Pieris rapae: addiction to glucosinolates? Entomologia Experimentalis et Applicata, Amsterdam, v. 91, p. 51-58, 1999.

ROESSINGH, P.; HORA, K. H.; FUNG, S. Y.; PELTENBURG, A.; MENKEN, S. B. J. Host acceptance behaviour of the small ermine moth Yponomeuta cagnagellus: larvae and adults use different stimuli. Chemoecology, Brussels, v. 10, p. 41-47, 2000.

SANTOS, G. P.; ZANUNCIO, J. C.; ZANUNCIO, T. V. Pragas do Eucalipto. Informe Agropecuário, Belo Horizonte, v. 9, p. 63-71, 1996.

SANTOS, G. P.; ZANUNCIO, T. V.; ZANUNCIO, J. C. Desenvolvimento de Thyrinteina arnobia Stoll (Lepidoptera: Geometridae) em folhas de Eucalyptus urophylla e Psidium guajava. Anais da Sociedade Entomológica do Brasil, Jaboticabal, v. 29, p. 13-22, 2000.

SCHENK, D.; BACHER, S. Functional response of a generalist insect predator to one of its prey species in the field. Journal of Animal Ecology, Oxford, v. 71, p. 524-531, 2002.

STADLER, E. Secondary sulphur metabolites influencing herbivorous insects. In BRUNOLD, S. Sulfur nutrition and sulphur assimilation in higher plants. Bern: P. Haupt, 2000. p. 187-202.

SZNAJDER, B.; HARVEY, J. A. Second and third trophic level effects of differences in plant species reflect dietary specialisation of herbivores and their endoparasitoids. Entomologia Experimentalis et Applicata, Amsterdam, v. 109, p. 73-82, 2003.

TRIGO, J. R. The chemistry of antipredator defense by secondary compounds in neotropical Lepidoptera: facts, perspectives and caveats. Journal of the Brazilian Chemical Society, São Paulo, v. 11, p. 551$561,2000$.

VLIEGER, L.; BRAKEFIELD, P. M.; MÜLLER, C. Effectiveness of the defence mechanism of the turnip sawfly, Athalia rosae (Hymenoptera: Tenthredinidae), against predation by lizards. Bulletin of Entomological Research, Farnham Royal, v. 94, p. 283-289, 2004.

VINSON, S. B. Parasitoid manipulation as a plant defense strategy. Annals of the Entomological Society of America, College Park, v. 92, p. 812-828, 1999. 
WEISER, L. A.; STAMP, N. E. Combined effects of allelochemicals, prey availability and supplemental plant material on growth of a generalist insect predator. Entomologia Experimentalis et Applicata, Amsterdam, v. 87, p. 181-189, 1998.

ZANUNCIO, J. C.; BATISTA, L. G.; ZANUNCIO, T. V.; VILELA, E. F.; PEREIRA, J. F. Levantamento e flutuação de lepidópteros associados à eucaliptocultura: VIII - Região de Belo Horiente, Minas Gerais, junho de 1989 a maio de 1990. Revista Árvore, Viçosa, M.G., v. 15, p. 83-93, 1991.

ZANUNCIO, J. C.; SANTOS, G. P.; BATISTA, L. G.; GASPERAZZO, W. L. Alguns aspectos da biologia de Dirphia rosacordis (Lepidoptera: Saturnidae) em folhas de eucalipto. Revista Árvore, Viçosa, M.G., v. 16, n. 1, p. 112-117, 1993.

ZANUNCIO, J. C.; NASCIMENTO, E. C. do; GARCIA, J. F. Major lepidopterous defoliators of eucalypt in southeast Brazil. Forest Ecology and Management, Amsterdam, v. 65, n. 1, p. 53-63, 1994.

ZANUNCIO, J. C.; TORRES, J.; LARANJEIRO, A. J. Biologia de Euselasia hygenius (Lepidoptera; Riodinidae) e seu consumo foliar em Eucalyptus urophylla. Revista Brasileira de Entomologia, São Paulo, v. 39, n. 3, p. 487-492, 1995. 